\title{
AFM Dissipation Topography of Soliton Superstructures in Adsorbed Overlayers
}

\author{
Carlotta Negri ${ }^{1}$, Nicola Manini ${ }^{1,2}$, Andrea Vanossi ${ }^{2,3}$, Giuseppe E. Santoro ${ }^{2,4}$, and Erio Tosatti ${ }^{2,4}$ \\ ${ }^{1}$ Dipartimento di Fisica, Università degli Studi di Milano, Via Celoria 16, 20133 Milano, Italy \\ ${ }^{2}$ International School for Advanced Studies (SISSA) and CNR-INFM \\ Democritos National Simulation Center, Via Beirut 2-4, I-34151 Trieste, Italy \\ ${ }^{3}$ CNR-INFM National Research Center S3 and Department of Physics, \\ University of Modena and Reggio Emilia, Via Campi 213/A, 41100 Modena, Italy \\ ${ }^{4}$ International Centre for Theoretical Physics (ICTP), Strada costiera 11, I-34151 Trieste, Italy
}

(Dated: January 6, 2010)

\begin{abstract}
In the atomic force microscope, the nanoscale force topography of even complex surface superstructures is extracted by the changing vibration frequency of a scanning tip. An alternative dissipation topography with similar or even better contrast has been demonstrated recently by mapping the $(x, y)$-dependent tip damping: but the detailed damping mechanism is still unknown. Here we identify two different tip dissipation mechanisms: local mechanical softness, and hysteresis. Motivated by recent data, we describe both of them in a one-dimensional model of Moiré superstructures of incommensurate overlayers. Local softness at "soliton" defects yields a dissipation contrast that can be much larger than the corresponding density or corrugation contrast. At realistically low vibration frequencies, however, a much stronger and more effective dissipation is caused by the tip-induced nonlinear jumping of the soliton, naturally developing bi-stability and hysteresis. Signatures of this mechanism are proposed for experimental identification.
\end{abstract}

PACS numbers: 46.55.+d, 07.79.Lh, 07.79.Sp, 81.40.Pq, 62.20.Qp

\section{INTRODUCTION}

The tip-based scanning force microscopes of the atomic force microscope (AFM) family constitute perhaps the single most important tool bag in nanotechnology. The substrate topography is extracted from a map of the oscillation frequency of a tip, hovering a short distance above the surface. Besides the frequency shift however, the tip also develops a damping, reflecting a position dependent mechanical dissipation. Maier et al. [1, 2] showed recently that AFM dissipation - whose general occurrence has been widely discussed by several groups a decade ago [3 7] but whose potential importance was still underestimated - is able to map exquisitely delicate features such as the Moiré superstructure pattern formed by misfit dislocations ("solitons") of incommensurate $\mathrm{KBr}$ adsorbate islands (Fig. 17) on $\mathrm{NaCl}(100)$. Surprisingly, the experimental dissipation map, Fig. 1b, showed similar or better contrast than the corresponding topographic map, with a characteristic reversed contrast (higher dissipation at the soliton, where topographic height is minimal [8]). Given also the great importance of achieving newer routes toward high-quality imaging, this is more than a mere curiosity, and deserves a proper understanding. Existing linear-response theory and other approaches to AFM dissipation 3, 9] and to general frictional dissipation [10 12] suggest a larger tip damping above softer substrates, and that provides an initial and valuable clue. Local tip dissipation can effectively reveal the underlying superstructure, since the local mechanical compliance is higher for example at surface soliton lines, where atoms sit at metastable positions. However at the relatively low AFM oscillation frequencies, the current understanding rules out linear response as the chief dissipation mechanism. A typical energy dissipation as large as $0.01-1 \mathrm{eV}$ per oscillation can only be accounted for by a hysteretic response of the interacting tip-substrate system, as was understood by theoretical analysis [13 15], and demonstrated experimentally [16, 17]. Such nonlinear effects of hysteresis are most likely involved in the surprisingly large AFM dissipation contrast of adsorbate superstructures too. Yet, it is unclear how inert systems (such as for example alkali halide overlayers) could give rise to hysteretic phenomena, and in particular how they would be connected to the presence of misfit superstructures ("solitons"). This is the issue which we address here by means of dynamical simulations of the simplest one-dimensional model. Our main result is the identification of an unexpected soliton-related hysteretic mechanism. During the first part of its swing, the tip can locally drag or push an underlying defect - here a soliton portion - causing it to jump across a (Peierls-Nabarro) energy barrier. During the return journey, the defect follows only sluggishly, and remains trapped somewhat longer on the wrong side of the energy barrier, thus opening a hysteresis loop. The area enclosed in the hysteretic force-displacement diagram represents a large tip energy dissipation, one that can survive down to realistically low AFM vibration frequencies, a regime where the linear-response dissipation is quantitatively irrelevant. This mechanism is likely to play a significant role every time a "softness pattern" is present, and should be easier to observe for horizontal than for vertical tip oscillations. 
(a)

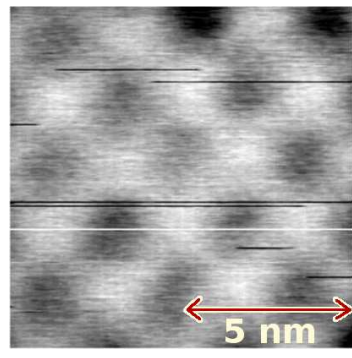

(b)

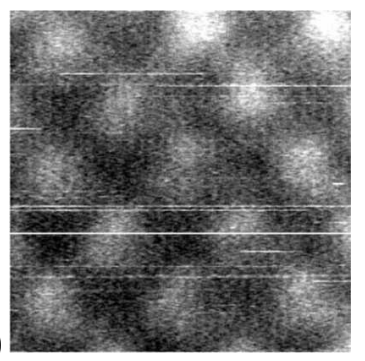

(c)

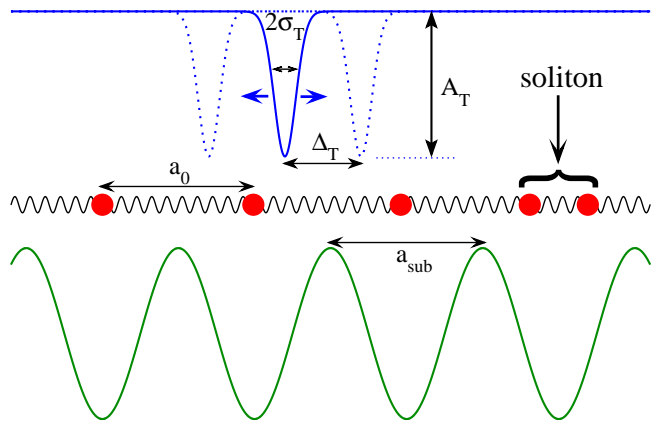

FIG. 1: (Color online) AFM Moiré superstructures of incommensurate $\mathrm{KBr}$ bilayers islands $/ \mathrm{NaCl}(100)$, adapted from Ref. [2]: (a) topography; (b) dissipation. Note the opposite phase: dissipation is largest at the soliton, where topographic height is minimal [8]. (c) $1 \mathrm{D}$ simulation model with a rigid substrate potential of period $a_{\mathrm{sub}}$, a harmonic chain of rest length $a_{0}$ (the adsorbate overlayer), and the localized tip potential $u_{\mathrm{T}}$ (here attractive) oscillating horizontally (in experiments the oscillation is usually vertical).

\section{THE MODEL}

To emphasize the basic and general aspects of the phenomena, rather than a realistic model targeted on the chemical detail of a specific tip/sample configuration 13 15, 18], we use the simplest possible model - a tip potential oscillating over a one-dimensional harmonic chain (the overlayer) moving in a rigid incommensurate periodic potential (the substrate), Fig. 16. The Hamiltonian of the mobile overlayer atoms is

$$
H=E_{k}+U_{\text {at-at }}+U_{\text {sub }}+U_{\mathrm{T}}(t),
$$

where $E_{k}=\frac{m}{2} \sum_{i} \dot{x}_{i}^{2}$ is the kinetic energy,

$$
U_{\text {at }-\mathrm{at}}=\frac{K}{2} \sum_{i}\left(x_{i+1}-x_{i}-a_{0}\right)^{2},
$$

is the mutual (harmonic) interaction potential, and $U_{\text {sub }}=\sum_{i} v\left(x_{i}\right)$ is the substrate potential, which we take of a pure cosine form:

$$
v(x)=-\frac{F_{\mathrm{sub}} a_{\mathrm{sub}}}{4 \pi} \cos \left(k_{\mathrm{sub}} x\right) .
$$

Here $a_{0}$ is the mean spacing between adatoms, $K$ is their mutual spring constant, $k_{\text {sub }}=2 \pi / a_{\text {sub }}$, and $a_{\text {sub }}$ is the period of the substrate potential [19, 20]. The two periodicities $a_{0}$ and $a_{\text {sub }}$ define the coverage $\theta=a_{\text {sub }} / a_{0}$. For convenience we take $a_{\text {sub }}$ as the unit length, $F_{\text {sub }}$ as the force unit, and the mass $m$ of the particles as the mass unit. (To get a feeling for quantities, the frequency units $F_{\text {sub }}^{1 / 2} m^{-1 / 2} a_{\text {sub }}^{-1 / 2}$ should be typical of an atomic vibration or a Debye frequency $\omega_{\mathrm{D}}$, typically $1 \mathrm{THz}$ or more). The general lack of commensuration between adsorbate and substrate periodicities gives rise to two-dimensional misfit dislocations, sometimes called solitons, which form a regular superstructure with the beat periodicity between the two. Fixed boundary conditions (BCs) are chosen in order to prevent the advancing tip to drag the entire pattern along, that would occur if, e.g., periodic BCs were used instead.

$U_{\mathrm{T}}(t)=\sum_{i} u_{\mathrm{T}}\left(x_{i}, t\right)$ is the time-dependent oscillating potential describing the tip action on the overlayer. We represent the AFM tip as a Gaussian-shaped oscillating potential, with $u_{\mathrm{T}}(x, t)=u\left(x-x_{\mathrm{T}}(t)\right), x_{\mathrm{T}}(t)=\bar{x}_{\mathrm{T}}+$ $\Delta_{\mathrm{T}} \cos \left(\omega_{\mathrm{T}} t\right)$, and

$$
u(x)=A_{\mathrm{T}} \exp \left(-x^{2} / \sigma_{\mathrm{T}}^{2}\right)
$$

Here $A_{\mathrm{T}}$ represents the repulsive (contact $\mathrm{AFM}, A_{\mathrm{T}}>$ 0 ) or attractive (noncontact AFM, $A_{\mathrm{T}}<0$ ) tip-atom interaction strength, $\sigma_{\mathrm{T}}$ is the tip width, $\Delta_{\mathrm{T}}$ and $\omega_{\mathrm{T}}$ are the tip oscillation amplitude and angular frequency around its central position $\bar{x}_{\mathrm{T}}$.

The equation of motion for the $i$-th overlayer atom is

$m \ddot{x}_{i}=-v^{\prime}\left(x_{i}\right)+K\left(x_{i+1}+x_{i-1}-2 x_{i}\right)+f_{\mathrm{T}}\left(x_{i}, t\right)-\gamma \dot{x}_{i}$,

where $v^{\prime}(x)=\frac{1}{2} F_{\text {sub }} \sin \left(k_{\mathrm{sub}} x\right)$, and the tip force

$$
f_{\mathrm{T}}(x, t)=-\frac{\partial}{\partial x} u_{\mathrm{T}}(x, t)
$$

is given by a straightforward analytical expression. A damping force term $-\gamma \dot{x}_{i}$ is introduced to represent all dissipation phenomena which remove energy and allow the attainment of a stationary frictional state.

We integrate the equations of motion (5) by means of a standard adaptive fourth-order Runge-Kutta routine 21] starting each simulation from a stationary fully relaxed overlayer, as obtained by a preliminary relaxation of equally-spaced adatoms $x_{i}(0)=i \cdot a_{0}$ and $\dot{x}_{i}(0)=0$. All simulations are carried out at a nearly commensurate coverage $\theta=1.06=\frac{53}{50}$, realized by means of a chain of $N=107$ particles in a region of length $L=100 a_{\text {sub }}$. A finite temperature $T$ is implemented by adding a standard Langevin random force to Eq. (5), and averaging over a long simulation time, usually at least 100 tip-oscillation periods. The extreme simplicity of the model allows us to extend simulations down to the realistic AFM frequency in the $\mathrm{MHz}$ range, which requires exceedingly long integration times. 


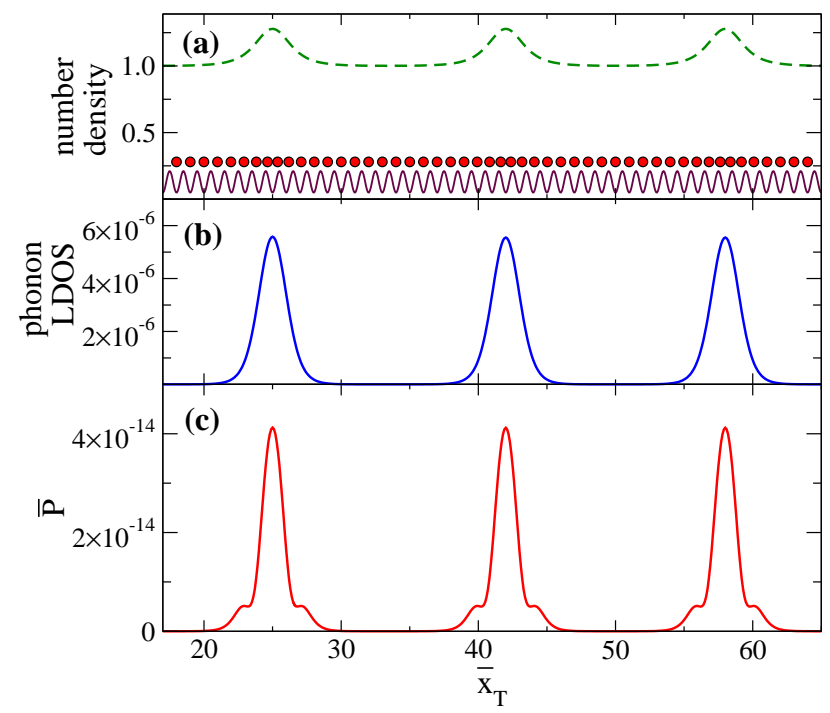

FIG. 2: (Color online) The linear-response regime. (a) Overlayer atom density (dashed), rest positions (circles), in the periodic substrate potential (solid). (b) The density of zerofrequency overlayer vibrational modes peaks at the soliton positions. (c) Dissipated power during tip vibration for a tip width $\sigma_{\mathrm{T}}=\Delta_{\mathrm{T}}=a_{\mathrm{sub}}$, weakly attractive potential of amplitude $A_{\mathrm{T}}=-10^{-4}, \gamma=0.2$ (underdamped regime), $K=5$ (fairly rigid overlayer), and oscillation frequency $\omega_{\mathrm{T}}=10^{-4} \pi$ (roughly $1 \mathrm{GHz}$ ). This regime is described well by linearresponse theory. Note a huge dissipation peak contrast at the soliton position relative to the terrace between solitons of about $10^{4}$.

The instantaneous power drained away by the damping term amounts to

$$
P_{\mathrm{diss}}=\sum_{i} \dot{x}_{i} \cdot\left(\gamma \dot{x}_{i}\right)=\gamma \sum_{i} \dot{x}_{i}^{2}=\frac{2 \gamma}{m} E_{c}
$$

and is thus proportional to the total kinetic energy of the overlayer. The power pumped by the tip into the chain is

$$
P_{\mathrm{T}}=\sum_{i} f_{\mathrm{T}}\left(x_{i}, t\right) \cdot \dot{x}_{i} .
$$

While these two quantities fluctuate separately, they must of course coincide on average over a period $\tau=$ $2 \pi / \omega_{\mathrm{T}}$ in the dynamical steady state

$$
\bar{P}=\frac{1}{\tau} \int_{\tau} d t P_{\mathrm{T}}(t)=\frac{1}{\tau} \int_{\tau} d t P_{\mathrm{diss}}(t),
$$

also indicating how the work done by the tip oscillation is eventually dissipated entirely by the viscous friction term.

\section{RESULTS: LINEAR RESPONSE AND BEYOND}

Figure 2 displays the dissipation results obtained in simulation for a weakly attractive tip potential, very high oscillation frequency, and general parameters that fall well inside the linear-response regime $[3,9,12]$. The linear response results show (i) strong dissipation enhancement at solitons, with $\bar{P}(\bar{x})$ several orders of magnitude stronger than in a terrace between two of them, closely mirroring the phonon local density of states (LDOS); (ii) dissipated power which is proportional to $A_{\mathrm{T}}^{2}$, independent of the attractive/repulsive sign of the tip-overlayer interaction, i.e. of the noncontact or contact mode of the AFM; (iii) absolute dissipation values that are very weak everywhere, and dropping with decreasing AFM frequency as $\left(\omega_{\mathrm{T}} / \omega_{\mathrm{D}}\right)^{2}$. Summing up, the predicted relative contrast of the soliton pattern in linear response dissipation is indeed very large. However, the exceedingly low value of realistic AFM frequencies $(\lesssim \mathrm{MHz})$ relative to microscopic frequencies $(\sim \mathrm{THz})$ renders this lineardissipation mechanism entirely academic.

We reach a more realistic regime by enhancing the tip-overlayer interaction strength, while still remaining in a moderate-interaction regime representing noncontact AFM. This new regime is dominated by nonlinear effects, where dissipation no longer drops as $\omega_{T}^{2}$, but at most linearly in $\omega_{\mathrm{T}}$ (apart from logarithmic corrections). Comparison of Fig. 3 3 with Fig. 2 2 shows that, nonlinear dissipation is again much larger near the solitons than in between them. A two-order of magnitude increase in $A_{\mathrm{T}}$ would in linear regime imply a dissipation increase by a factor $10^{4}$, whereas we find a much larger factor of about $10^{6}$ already at this large frequency $\left(\omega_{\mathrm{T}}\right.$ is here $10^{-4} \pi$, corresponding to the gigahertz range). Decreasing frequency down to realistic AFM values, the increase will become gigantic, because the nonlinear dissipation lacks the extra power of $\omega_{\mathrm{T}}$ appearing in the linear-regime dissipation. The new element brought in by nonlinearity is mechanical. A strongly interacting tip is now able to drag, or to push, the soliton - a mobile entity - forward or backward during the oscillation cycle. As the soliton must overcome the (Peierls-Nabarro) barrier in order to move, its motion is sluggish, and can follow the tip only with hysteresis and, as anticipated, hysteresis entails a large dissipation. As shown in detail in Fig. 3a,b, the higher dissipation point A is found to corresponds to two successive Peierls-Nabarro barriers being overcome in the oscillatory process, the smaller dissipation point $\mathrm{B}$ to a single barrier. The dissipation at point $\mathrm{C}$, where the tip potential is unable to "grab" the soliton, is negligible by comparison. The onset of this large-dissipation region, dominated by hysteresis, is rather sharp. Fig. (4)illustrates this point, by showing the average power $\bar{P}$ at location A, divided by the linear-regime factor $\left|A_{\mathrm{T}}\right|^{2}$, as a function of the tip amplitude $\left|A_{\mathrm{T}}\right|$. Beyond a value of $\left|A_{\mathrm{T}}\right|$ of order $4 \times 10^{-3}$, the linear regime behavior is abruptly abandoned, and the dissipation increases rapidly by several orders of magnitude. 

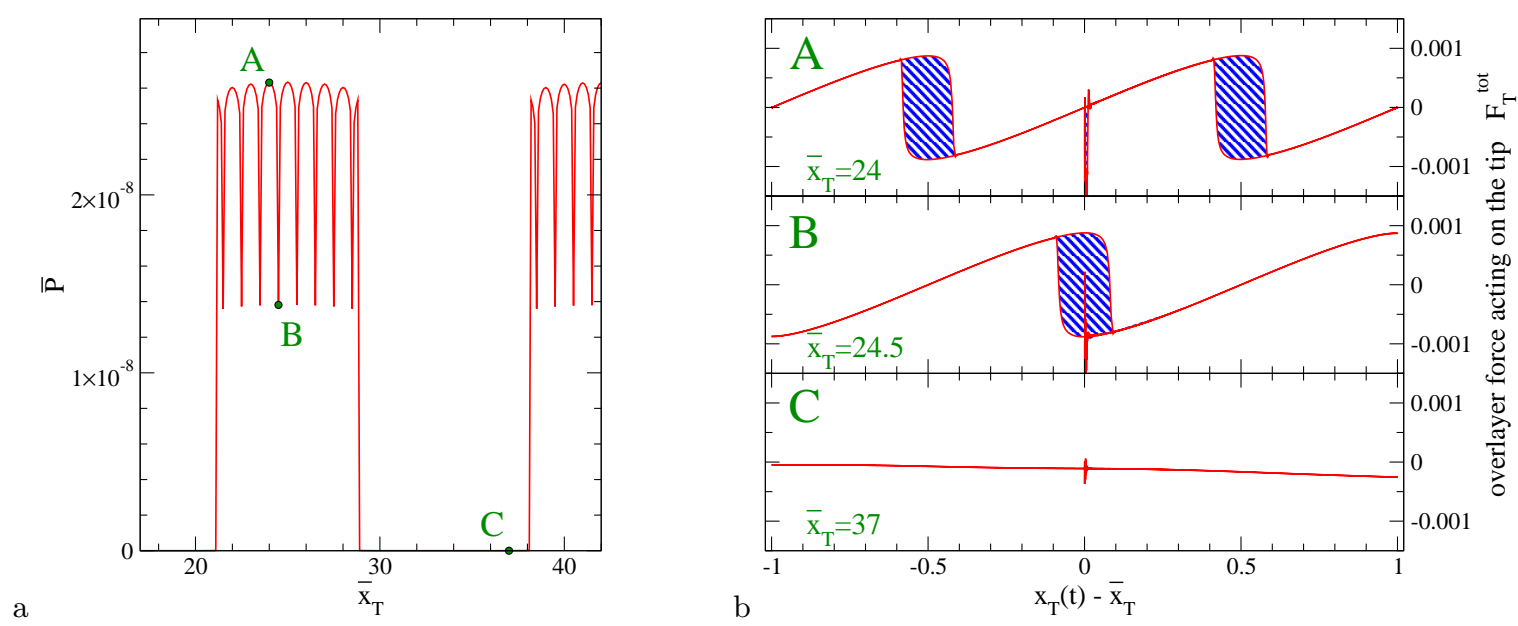

FIG. 3: (Color online) The strong-interaction hysteretic regime. (a) Mean power dissipated in the steady regime by a strongly interacting tip $\left(A_{\mathrm{T}}=-0.01\right.$, all other parameters the same as in Fig. 2) scanning the same overlayer $(K=5)$. (b) Forcedisplacement response at three typical scanning points marked in the left panel. The strong dissipation at the solitons is now due to hysteretic jumps of the solitons (kinks) across their Peierls-Nabarro barriers. The dissipated energy in a cycle equals the area of the hysteretic loop in the force-displacement plane (shaded). When the potential is strong enough to drag or push a soliton (points A, B), this occurs with hysteresis and the dissipation is large. With the selected oscillation amplitude, the soliton is dragged across two barriers (point A) or a single barrier (point B), depending on the center of tip position $\bar{x}_{\mathrm{T}}$. When the tip grabs no soliton (point $\mathrm{C}$ ) there is no hysteresis and dissipation drops.

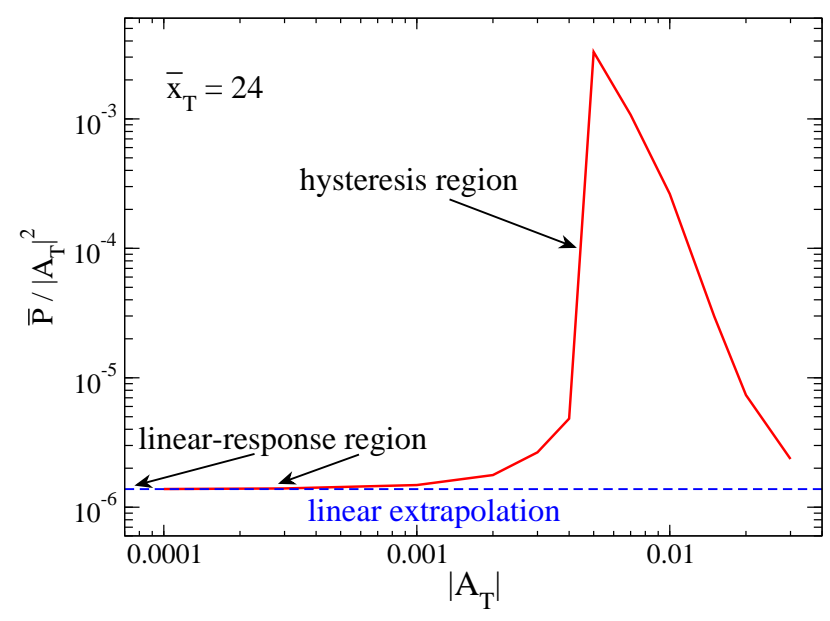

FIG. 4: (Color online) The dissipated power normalized to the linear regime scaling, $\bar{P} /\left|A_{\mathrm{T}}\right|^{2}$, versus the strength of the tip-overlayer interaction $\left|A_{\mathrm{T}}\right|$, at point A of Fig. 3 and for the same parameters as in Fig. 2 Notice the sharp onset of the non-linear regime where the hysteretic mechanisms starts to play a role, leading to a strong enhancement of dissipation.

\section{PREDICTIONS AND DISCUSSION}

Our simulated example strongly suggests that a large hysteretic component should be present in the existing dissipation maps 22] of Moiré patterns. More generally, hysteretic defect dragging should dominate the AFM dissipation maps. What are the predicted signatures of this mechanism? Our model study suggests two main signatures.

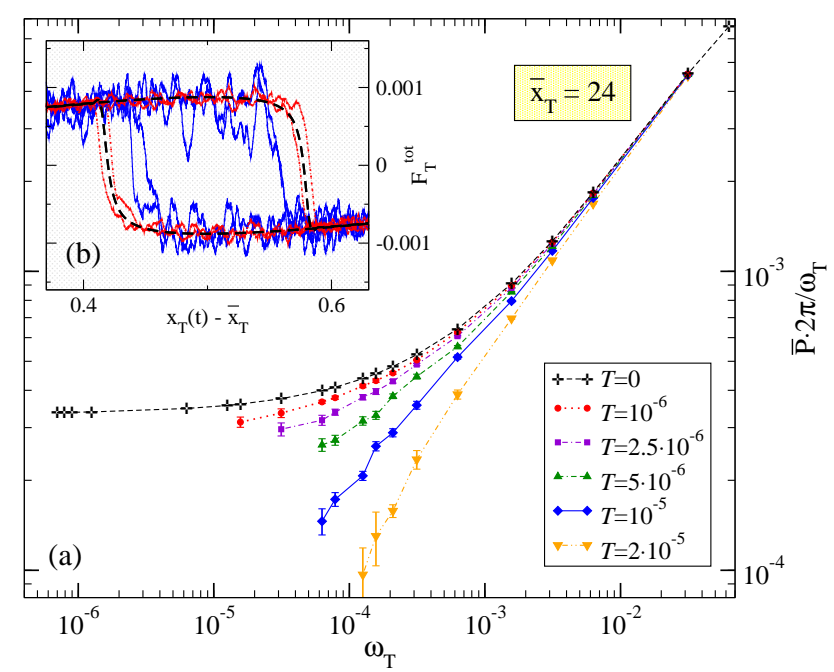

FIG. 5: (Color online) The dissipation reduction due to thermal shrinking of the hysteretic loop, for $\omega_{\mathrm{T}}=10^{-4} \pi$ at the soliton location A of Fig. 3 (a) The frequency dependence of the energy dissipated in one period $\bar{P} 2 \pi / \omega_{\mathrm{T}}$, computed for several temperatures, exhibiting a clear reduction of $\bar{P} 2 \pi / \omega_{\mathrm{T}}$ due to a rise in temperature. (b) Detail of the right-side area of the force-displacement dependency following two typical tip-oscillation periods for $T=10^{-5}$, solid, and $10^{-6}$, dotted, compared to $T=0$, dashed: the hysteretic area is shrinking due to a randomly anticipated thermally activated barrier crossing.

(i) Abruptness of AFM friction onset with increasing strength of tip-surface interaction. As suggested by Figs. 3,4, dragging sets in abruptly only above a cer- 
tain threshold, which means below a certain tip-surface or tip-soliton distance.

(ii) Anomalously mild (linear with logarithmic corrections) frequency dependence of AFM friction at finite temperature. It is a rather general property of all hysteretic friction phenomena to heal away at sufficiently low frequencies, where adiabatic motion allows sufficient time to jump thermally over barriers. For instance, thermolubricity experiments [23 26] and detailed calculations within the Tomlison model [27] show an average friction force $F=F_{c}-A T^{2 / 3}|\log (\alpha v / T)|^{2 / 3}$, where $v$ is the driving velocity, and $A$ and $\alpha$ are system-dependent dimensional constants. In incommensurate overlayers, the soliton nearest to the tip behaves similarly to a Tomlinson particle, as is driven across a Peierls-Nabarro barrier. In Fig. 5a we do observe a thermal reduction of dissipation, due to a shrinkage of the hysteretic loop, illustrated in Fig. 5b. When driving is oscillatory as in AFM dissipation, the role of $v$ is taken by $\Delta_{\mathrm{T}} \omega_{\mathrm{T}}$. We find our data to be compatible with a similar relation $\bar{P}(T)=\bar{P}(0)-A^{\prime} T^{2 / 3}\left|\log \left(\alpha^{\prime} \omega_{\mathrm{T}} / T\right)\right|^{2 / 3}$. The parameters $A^{\prime}$ and $\alpha^{\prime}$ are here related to the effective soliton properties (mass, damping, barrier height...), and are nontrivial functions of the "bare" model parameters.

We conclude that AFM dissipation maps of incommensurate overlayer superstructures can in principle achieve an extremely high contrast resolution of soliton defects relative to commensurate terraces. The most important theoretically predicted dissipation mechanism is the nonlinear dragging or pushing of some local portion of the defect, where the large tip damping is associated with hysteresis of defect motion. Besides a sharp threshold in the tip-surface interaction and oscillation magnitudes, this mechanism predicts a very characteristic logarithmic dependence (eventually turning to linear at extremely low frequencies) of dissipation upon frequency and temperature. More generally, the nonlinear dragging of soft defects or features (e.g. a floppy residue in a biomolecule) should give rise to a strong visibility in AFM dissipation topography, of considerable potential impact for applications.

This work was supported by CNR, as a part of the European Science Foundation EUROCORES Programme FANAS.

[1] S. Maier, E. Gnecco, A. Baratoff, R. Bennewitz, and E. Meyer, Phys. Rev. B 78, 045432 (2008).

[2] S. Maier, O. Pfeiffer, Th. Glatzel, E. Meyer, T. Filleter, and R. Bennewitz, Phys. Rev. B 75, 195408 (2007).

[3] M. Gauthier and M. Tsukada, Phys. Rev. Lett. 85, 5348
(2000).

[4] R. Bennewitz, A. S. Foster, L. N. Kantorovich, M. Bammerlin, C. Loppacher, S. Schär, M. Guggisberg, E. Meyer, and A. L. Shluger, Phys. Rev. B 62, 2074 (2000).

[5] C. Loppacher, R. Bennewitz, O. Pfeiffer, M. Guggisberg, M. Bammerlin, S. Schär, V. Barwich, A. Baratoff, and E. Meyer, Phys. Rev. B 62, 13674 (2000).

[6] C. Loppacher, M. Bammerlin, M. Guggisberg, S. Schär, R. Bennewitz, A. Baratoff, E. Meyer, and H.-J. Güntherodt, Phys. Rev. B 62, 16944 (2000).

[7] P. M. Hoffmann, S. Jeffery, J. B. Pethica, H. Özgür Özer, and A. Oral, Phys. Rev. Lett. 87, 265502 (2001).

[8] J. Baker and P.-A. Lindgard, Phys. Rev. B 54, R11137 (1996).

[9] M. Gauthier and M. Tsukada, Phys. Rev. B 60, 11716 (1999).

[10] E. Granato and S. C. Ying, Phys. Rev. B 59, 5154 (1999).

[11] B. N. J. Persson, Sliding Friction: Physical Principles and Applications (NanoScience and Technology) (Springer-Verlag, Berlin 1998).

[12] B. N. J. Persson, E. Tosatti, D. Fuhrmann, G. Witte, and Ch. Wöll, Phys. Rev. B 59, 11777 (1999).

[13] L. N. Kantorovich and T. Trevethan, Phys. Rev. Lett. 93, 236102 (2004).

[14] T. Trevethan and L. Kantorovich, Nanotechnology 17, S205 (2006).

[15] S. A. Ghasemi, S. Goedecker, A. Baratoff, T. Lenosky, E. Meyer, and H. J. Hug, Phys. Rev. Lett. 100, 236106 (2008).

[16] A. Schirmeisen and H. Hölscher, Phys. Rev. B 72, 045431 (2005).

[17] R. Hoffmann, A. Baratoff, H. J. Hug, H. R. Hidber, H v Löhneysen, and H.-J. Güntherodt, Nanotechnology 18, 395503 (2007).

[18] V. Caciuc, H. Hölscher, D. Weiner, H. Fuchs, and A. Schirmeisen, Phys. Rev. B 77, 045411 (2008).

[19] O. M. Braun and Yu. S. Kivshar, The Frenkel-Kontorova Model: Concepts, Methods, and Applications (SpringerVerlag, Berlin, 2004).

[20] A. Vanossi and O. M. Braun, J. Phys.: Condens. Matter 19, 305017 (2007).

[21] W. H. Press, S. A. Teukolsky, W. T. Vetterling and B. P. Flannery, Numerical Recipes in Fortran. The Art of Parallel Scientific Computing (Cambridge University Press, Cambridge, 1996).

[22] B. J. Albers, T. C. Schwendemann, M. Z. Baykara, N. Pilet, M. Liebmann, E. I. Altman, and U. D. Schwarz, Nature Nanotech. 4, 307 (2009).

[23] E. Gnecco, R. Bennewitz, T. Gyalog, Ch. Loppacher, M. Bammerlin, E. Meyer, and H.-J. Güntherodt, Phys. Rev. Lett. 84, 1172 (2000).

[24] E. Riedo, E. Gnecco, R. Bennewitz, E. Meyer, and H. Brune, Phys. Rev. Lett. 91, 084502 (2003).

[25] E. Gnecco, R. Bennewitz, A. Socoliuc, and E. Meyer, Wear 254, 859 (2003).

[26] S. Sills and R. M. Overney, Phys. Rev. Lett. 91, 095501 (2003).

[27] Y. Sang, M. Dubé, and M. Grant, Phys. Rev. Lett. 87, 174301 (2001). 